\title{
PERVASIVE SOFTWARE SERVICES FOR DYNAMIC VIRTUAL ORGANIZATIONS
}

\author{
Schahram Dustdar and Harald Gall \\ Distributed Systems Group, Vienna University of Technology \\ Argentinierstrasse 8/184-1, 1040 Wien, AUSTRIA \\ [dustdar, gall]@infosys.tuwien.ac.at
}

\begin{abstract}
Information and Communication Technology (ICT) is penetrating deeper into daily life and work of people: mobile communications, portable devices, embedded computing and intelligent systems are major opportunities for creating next generation information systems and infrastructures. Technologies such as Bluetooth, WLAN, or SIP-based broadband networks and 3rd generation mobile phones (UMTS) are offering the infrastructure to conceive information systems as pervasive, meaning that systems are accessible from anywhere, at any time, and with any device. This paper presents our achievements in this area of research based the research prototypes developed in our EU projects MOTION and EasyComp. Further, the paper outlines aspects of pervasive software services for dynamic virtual organizations that are considered in our ongoing project PeSSIFIC to fulfill needs and constraints of distributed and mobile collaboration scenarios.
\end{abstract}

\section{INTRODUCTION}

Pervasiveness is an important means to support new business models and encourage new ways of work. Recent developments show a strong move towards increasingly mobile nimble and virtual (project) teams. Whereas traditional organizational structures relied on teams of collaborators dedicated to a specific project for a long period (Classic Teams, see Figure 1), many organizations increasingly rely on nimble teams, formed from members of possibly different branches or companies, assigned to perform short-lived tasks in an ad-hoc manner. For team members, tasks may be small parts of their overall work activities. Such nimble collaboration styles change many of the traditional assumptions about teamwork: collaborators do not report to the same manager, they do not reside in the same location, and they do not work during the same time. As a consequence, the emerging new styles of distributed and mobile collaboration across organizational boundaries are fostering new interaction patterns of working which can be characterized as follows:

- Nimble teams ( $N$-teams) represent a short timeframe of team constellations that emerge, engage in work activities, and then dissolve again, as the circumstances require. 
- Virtual project teams (V-teams) require and enable people to collaborate across geographical distance and professional (organizational) boundaries and have a somewhat stable team configuration with roles and responsibilities assigned to team members.

- Nomadic teams (M-teams) allow people working from home, on the move, or in flexible office environments, and any combinations thereof.

N/V/M-teams share the notion that they work on common goals, whereby work is being assigned to people in terms of objectives, not tasks. This new way of collaboration leads to challenging and new requirements with respect to the software infrastructure. In our EU project MOTION we have investigated that a goal-oriented working style requires pro-active software service aggregation and provisioning. Basic information technology to support teamwork has enabled sharing of information (databases) and communication (electronic mail). More recently, chat software has enabled synchronous communication. Many commercial and research systems have been built to support teams. Most, if not all, of these are closed environments that require all users to operate within the system. While such solutions may work for traditional teams that work within one organization and the manager can mandate the use of particular software tools and environments, with ad hoc teams a closed system is not possible. Different people, coming from different organizations will have their own preferences and experiences and cannot be expected to undergo a long learning cycle before participating in team activities. Certainly, a team that is formed to solve a short-lived problem is not able to go through a long deliberation cycle to select a teamwork support environment.

The new interaction patterns of various team configurations increasingly require software provided as services on different devices and independent of location. To enable these scenarios, a new wave of pervasive software services will exploit the knowledge developed and deployed for conventional information systems, but will also need advanced concepts, models, methodologies, and supporting technologies to fully exploit the potentials of the enabling infrastructure. First and foremost a significant investigation in the hardware and software infrastructure is required and presented in detail in this paper. These considerations constitute a significant entry barrier for research in this field. This creates a need for pervasive software service infrastructures that enable flexible and mobile collaboration.

The goal of our research project is to build a pervasive software service infrastructure, which facilitates N/V/M team configurations, goals and objectives, and enables powerful and flexible ways of interaction models by pro-actively aggregating software services (using message-oriented middleware and Web services as well as peer-to-peer systems) and utilizing new network technologies such as Bluetooth and WLAN on many devices (Personal Digital Assistants, SmartPhones, Tablet PCs, Notebooks etc.) over high-speed SIP-based broadband networks.

\section{SHORTCOMINGS OF CURRENT APPROACHES}

Current collaboration systems such as Workflow Management Systems (WfMS) focus on automating structured (modeled) intra-organizational business processes. 
Groupware (Dayal et al., 2001; Ellis et al.; 1991), on the other hand, typically does not contain any knowledge or representation of the goals or underlying business processes of the group (Schal, 1996; Ellis, 1991). There have been considerable attempts to merge workflow-, groupware-, and business process modeling technologies (Dayal, 2001). Future collaboration systems supporting distributed and mobile collaboration need to focus on covering inter-organizational activities and processes including product value-chains on the Internet (Schal, 1996) regardless of location (mobility) and regardless of devices used.

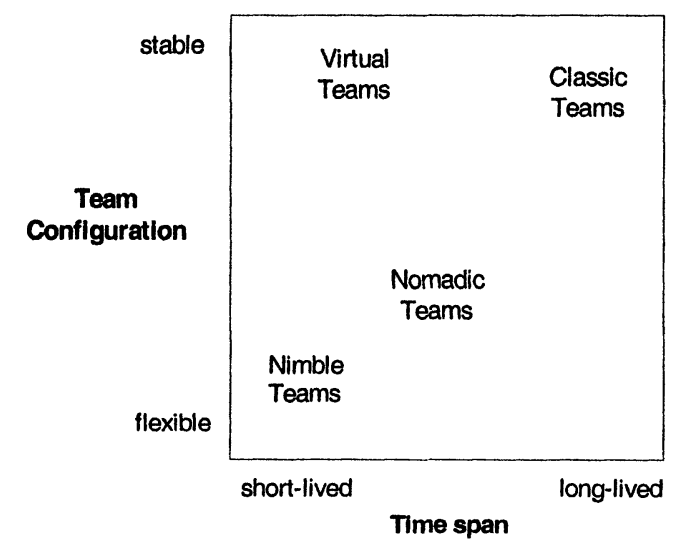

Figure 1 - Team aspects along configuration and time dimensions

Current WfMS and Groupware systems do not combine the features that N/V/M teams require: pro-active service composition and aggregation, information sharing, process-sharing, -composition, and -configuration. N/V/M-teams have the following main characteristics: loose coupling, decentralized and flexible control of business processes, resources (e.g. services and storage), their objectives, goals and tasks. Furthermore, minimal global knowledge exists regarding where, when, and how particular servers and services are accessible, since autonomous nodes that can join and leave N/V/M-teams or communities any time wanted or per request. These characteristics can be well supported by the integration of peer-to-peer $(P 2 P)$ middleware and Web services principles addressed in our project "Pervasive Software Services Infrastructure for Collaboration (PeSSIFIC)."

As a consequence, both data and service-oriented P2P aspects must be combined and pro-active as well as reactive mechanisms that support the new interaction patterns among computing nodes and N/V/M-team members must be devised. The definition, composition, and coordination of services and data offered by peers (i.e. computing nodes) should enable dynamic and adaptive configurations of teams and project (process) resources to exchange arbitrary kinds of data. Virtual information spaces should be facilitated to set-up, configure, adapt, and maintain for the above mentioned interaction patterns, thus enabling new ways of working, which can be characterized as follows:

- Virtual Project Teams: people collaborating across geographical distance and professional (organizational) boundaries 
- Common Goal working: work being assigned to people in terms of objectives, not tasks

- Nomadic working: people working from home, on the move, or in flexible office environments

- Nimble Teams: short timeframe of work group constellations that emerge, engage in activities, and then dissolve again, as the circumstances require.

Pervasive technologies will allow people to work in a more flexible way, having supporting tools available anytime, anywhere, rather than being constrained to a specific location.

\section{RESEARCH APPROACH}

An important objective of our research is to build a pervasive software services infrastructure for collaboration for N/V/M-teams utilizing various devices (PDAs, SmartPhones, Tablet PCs, Notebooks) and network technologies (Bluetooth, WLAN) as well as notification services (e.g. publish/subscribe to syndicate content including video and audio). The goal of this paper is to present the scientific foundations and their innovative set of methodologies, techniques and tools for supporting the modeling, definition, composition, and distributed enactment (orchestration) of software services to support multiple interaction patterns for distributed and mobile collaboration. The research goals are:

- An open software architecture that defines the components, connectors and configurations required for pervasive software services infrastructure for collaboration.

- An open software platform realizing the architectural components in a research prototype implementation.

- Framework of dynamic composition of (complex) pervasive software services to enable process awareness for loosely coupled collaboration scenarios (e.g. common goal working for N/V/M-teams).

- Generally applicable pro-active and reactive communication and coordination models for N/V/M-teams.

- Quality-of-service models to support informed decisions based on QoS parameters (provision of "best available" service, negotiation of service levels, etc.).

- Techniques and methodologies for building up ad-hoc storage systems and decentralized data placement strategies for optimizing data distribution and replication to support N/V/M-team scenarios.

- Decentralized trust and access control models that enable risk assessment of diverse interaction styles and protection of resources.

- Influence of imperfect access links on performance and reliability of pervasive software services

Although access networks only provide access to specific pervasive software services, the properties of the used access links have a strong influence on the performance and usability of different software services. For example a slow data 
rate Bluetooth link can probably not be used for video streaming whereas Wireless LAN could support such a service. This means that not all access technologies are suitable for specific software services.

Apart from the theoretical performance of a utilized access technology, the actual performance of it depends on many aspects like the current link quality in case of GPRS or the interference situation for WLAN (e.g. WLAN with Bluetooth interference). This means that in reality one has to deal with imperfect access links, which can lead to significantly reduced performance of the considered access technology and therefore reduced applicability for specific software services.

Mobility is another important aspect regarding pervasive software services. Whereas in current mobile applications mobility typically means to stay within range of a chosen access technology, future applications are likely to utilize different access networks within one session. This means that seamless mobility between access networks will be required. The properties and imperfections of such "mobility" influence also the performance and usability of pervasive software services.

\section{CURRENT STATE AND PROTOTYPE}

The current state-of-the-art in collaboration systems exhibits serious limitations that hinder the diffusion of technologies for teams requiring new interaction styles into pervasive software services for collaborative work. The approach of this project exhibits several distinguishing characteristics: (a) architecture-driven: the software architecture considerations and concerns play a major role in the development of methodologies, techniques, and tools; (b) well-grounded in results of our EUprojects MOTION ${ }^{1}$ and EASYCOMP ${ }^{2}$; and (c) prototype-oriented in that it will deliver prototype tools as proof-of-concept. These shortcomings will be dealt with some technologies elaborated in our IST EU-research projects MOTION and EasyComp listed below. New challenges of pervasive software services for dynamic virtual organizations are currently being implemented in our PeSSIFIC project, outlined below.

In MOTION we have designed and implemented a teamwork services platform that offers the following software services for dynamic virtual organizations: 1) support for publish/subscribe and event-based software services for collaboration; 2) mobility enabling information tailoring and device-independent representation; 3 ) process awareness for loosely coupled workflows for N/V/M-teams; 4) structured meta-data for multiple kinds of resources (team members, artifacts, processes) that are accessible for complex queries and retrievals; 5) authorization and trust models defining who is allowed to access data and services; 6) structured meta-data for multiple kinds of resources (team members, artifacts, processes) that are accessible

1 The MOTION project consortium consisted of the following partners: Nokia Research Center, Finland; Politecnico di Milano, Italy; Softeco, Italy; Zanussi-Electrolux, Italy; and Vienna University of Technology, Austria.

2 The EASYCOMP (Easy Composition) project consortium consisted of the following partners: Linkoepings University,

University of Karlsruhe, University of Kaiserslautern, ARM-DI Ecole des Mines Nantes, Twente University, Budapest University Institute of Technology, HEI Informationssystems, QLABS France, ILOG SA, and Vienna University of Technology, Austria. 
for complex queries and retrievals; and 7) authorization and trust models defining who is allowed to access data and services.

From the EasyComp project we adopt mainly component technologies that support the composition of components across different component models and allow component inspection and adaptation for different software environments to announce them as software services in a pervasive environment.

For PeSSIFIC we base upon the above technologies and the developed prototype, but in order to fulfill our ambitious goals for distributed and mobile collaboration with pervasive software services, we are integrating and enhancing our previous results with the following technologies:

- Pro-active software service composition for nimble teams (value added service aggregation based on goals and objectives)

- Provisioning of pervasive software service infrastructure for collaboration utilizing Message-oriented Middleware (MoM), Web Services, and Session Initiation Protocol (SIP)-Servers

- Enabling and supporting common goal oriented working for N/V/M-teams

- Dynamic combinations of data and services as well as complex interactions (e.g. reactive interactions)

- Content placement and syndication infrastructure (including video streaming for collaboration)

- Decentralized and dynamic control and management of resources for new interaction patterns

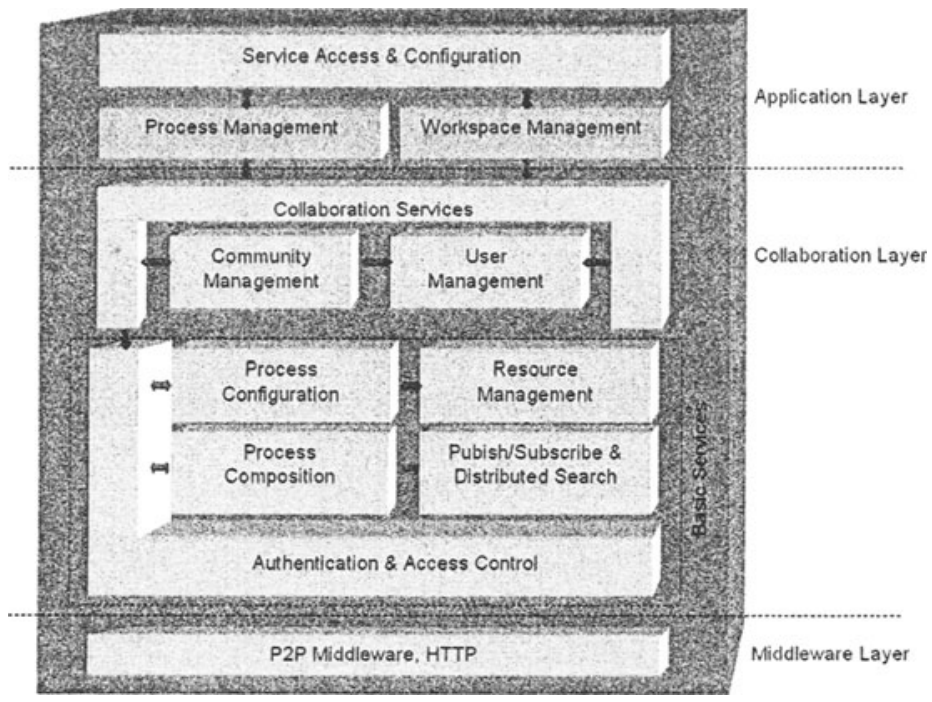

Figure 2-PeSSIFIC Conceptual Architecture

This PeSSIFIC project follows the conceptual software architecture described in our previous work (Dustdar et al., 2002a; Dustdar et al., 2002b; Dustdar et al., 2003; Kirda et al., 2001) and refines this initial architectural approach (shown in Figure 2). 
The project covers major aspects of such an architecture targeting functionalities and properties of each of the sketched layers (application, collaboration, middleware layers). The project will enhance the MOTION prototype and additionally build demonstrator applications; these are small applications but have enough functionnality to be a convincing proof-of-concept.

The PeSSIFIC platform will support the following key aspects:

- mobility of users to support multiple connectivity modes (connected, disconnected, ad-hoc)

- data sharing in virtual project communities

- distributed searches and data delivery

- business process support

In technical terms the approach is based on an open distributed software architecture including peer-to-peer, Web services, and workflow, publish-subscribe for notifycation and updating of users about artifacts and processes, process configuration, composition and enactment for flexible design and formation of emerging interaction patterns, and pervasive software services and components to enable multiple team configurations.

From a hardware perspective (see Figure 3) the Pervasive Software Service Infrastructure comprises 4 servers with fiber channel host bus adapters (HBA) and a Storage Area Network (SAN). Three servers are equipped with message oriented middleware (MoM) from IBM (WebSphere MQ) for publish/subscribe and eventbased mechanisms and service modeling software from Microsoft. One server is equipped with a SIP-server (Session Initiation Protocol).

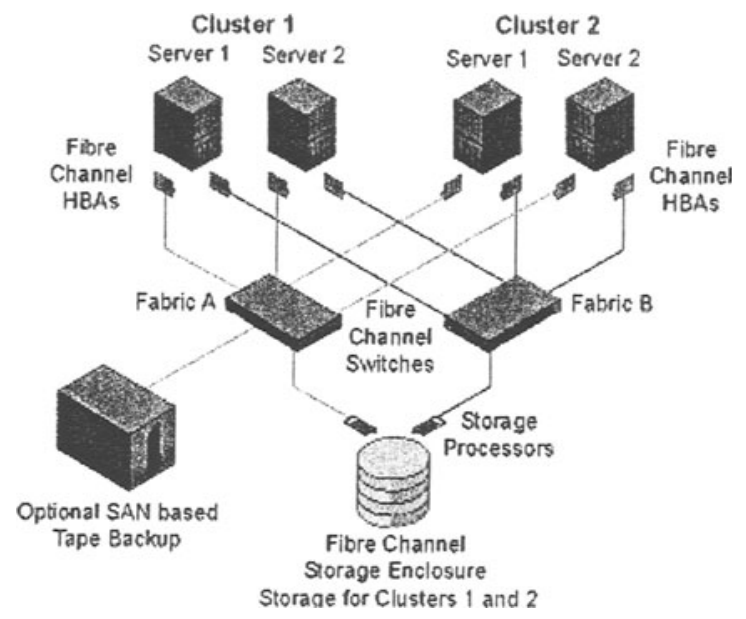

Figure 3 - Hardware for Pervasive Software Service Infrastructure

The SIP-server is responsible for managing and initiating collaborative sessions from team members, based on device capability - and capacity modes (e.g. Audio, Video, Pocket PC, Notebook, SmartPhone, Tablet PC etc.), whereas the MoM servers are responsible for handling subscriptions for team members. 
Events and subscriptions of team members are of high volume and strongly dependent on the communications type and team configurations (nimble-, virtual-, and nomadic teams). This infrastructure should be able to handle the required high load of asynchronous messages being exchanged for dynamic pervasive software service composition and execution as well as initiating synchronous multimedia communications such as audio/video conferencing and streaming, and chat-sessions thereby utilizing the SIP-server. Validation of the proposed infrastructure is currently performed such that more detailed results on the behavior can be reported soon.

\section{CONCLUSIONS}

Pervasive software services are an essential requirement for distributed and mobile collaboration in dynamic organizations, crossing organization and location boundaries of people. In this paper we have presented our achievements in this area of research based the research prototypes developed in our EU projects MOTION and EasyComp. For that, we have outlined components and software layers for pervasive software services in dynamic virtual organizations that are considered in our ongoing project PeSSIFIC: software services for teams such as expertise sharing and community building; a middleware platform providing peer-to-peer communication, messaging, virtual information spaces, publish-subscribe functionality, combined with (business) process composition, configuration and enactment components; and a hardware infrastructure including SANs, MoM and session management over SIP.

Currently the software and hardware infrastructure is being evaluated and tested in our DMC lab environment to get more insights about necessary improvements and enhancements.

\section{REFERENCES}

1. Dayal U, Hsu M, Ladin R. Business Process Coordination: State of the Art, Trends, and Open Issues. In Proc. of the 27th VLDB Confererence, Roma, Italy, 2001.

2. Dustdar S, Gall H. Architectural concerns in distributed and mobile collaborative systems. Proc. of the 11-th Euromicro Conference on Parallel Distributed and Network based Processing (PDP 2003), Genoa, Italy, February 2003.

3. Dustdar S, Gall H. Process Awareness for distributed software development in virtual teams. 28th EUROMICRO Conference: Software Process and Product Improvement, September, IEEE Computer Society Press, 2002.

4. Dustdar S, Gall H. Mobile and Distributed Collaboration in Virtual Communities. Proceedings of IFIP Conference on Infrastructures of Virtual Enterprises, Portugal, Kluwer Academic Publishers, May 2002.

5. Ellis CA, Gibbs SJ, Rein GL. Groupware: some issues and experiences. Communications of the ACM, 34, 1, 39-58, 1991.

6. Kirda, E., G. Reif, P. Fenkam, H. Gall, G.P. Picco, G. Cugola. A Web-based peer-to-peer architecture for collaborative nomadic working, 10th IEEE Workshops on Enabling Technologies: Infrastructures for Collaborative Enterprises (WETICE), Boston, MA, USA, June 2001.

7. Puustjărvi, J., H. Laine. Supporting cooperative inter-organizational business transactions. In Proc. DEXA 2001, Computer Science Lecture Notes, Springer Verlag, pp. 836-845, 2001.

8. Schal, T. Workflow Management Systems for Process Organizations. New York: Springer, 1996. 